\title{
Torque Gap Filler for Automated Manual Transmissions: Principles for the Development of the Control Algorithm
}

\author{
Aldo Sorniotti \\ University of Surrey, United Kingdom
}

Copyright $\odot 2009$ SAE International

\begin{abstract}
One of the most significant tasks in automotive design is related to the implementation of gearboxes capable of reducing the torque gap during the gearshift process and, at the same time, not decreasing vehicle performance from the point of view of driveline efficiency. Automated gearboxes based on torque converters ([1], [2]) satisfy the first requirement but not the second. On the other hand, manual automated gearboxes ([3], [4], [5], [6]) satisfy the requirements in terms of consumption, due to the absence of the dissipations caused by the torque converter. In fact, they consist of the basic layout of a manual transmission with hydraulic or electromechanical actuators which are adopted for the clutch and the synchronizers. However, automated manual transmissions cannot guarantee optimal longitudinal dynamics of the vehicle due to the discontinuity in torque transmission when the clutch is disengaged. New solutions are based on the adoption of Dual Clutch systems ([7], [8], [9]) characterized by a significant mechanical complexity. This paper presents, from the points of view of basic concepts and vehicle dynamics simulation, the Torque Gap Filler, a new system patented by the author ([10], [11]) capable of joining the advantages of the systems described in [1] - [9] without their disadvantages. First of all, the basic concepts of the system are described in detail. Then the basic equations necessary for the development of the control algorithm of the system are presented, and finally a discussion about the possible benefits of the system for two different mechanical layouts are discussed.
\end{abstract}

\section{SYSTEM LAYOUT}

Torque Gap Filler (TGF) is a system which can be added to the conventional layout of a manual gearbox, without a significant variation to the external size of the system. From a practical point of view, it could be implemented by adopting the two distinct physical configurations presented in Figures 1 and 2. The basic concept of TGF consists of connecting, during gearshift, the engine shaft and the secondary shaft of a manual gearbox through an epicyclical gearbox. The three elements of the epicyclical group which are connected with the rest of the driveline are the sun gear, the ring gear and the carrier. One of these elements is connected to the engine flywheel, another one to the secondary shaft of the gearbox and the third one to a hydraulic brake, which (if activated) permits the transmission of the torque to the secondary shaft of the gearbox. In configuration 1 (Figure 1), the flywheel is connected to the sun gear and the brake to the ring gear. In configuration 2 (Figure 2), the flywheel is connected to the ring gear and the brake is connected to the sun gear.

When the driveline is not performing a gearshift, the brake is released and the components of the epicyclical gearbox transmit zero torque (with the exception of the friction losses), depending on the inserted gear ratio. The brake has to be properly activated when the vehicle performs an upshift. A possible target for the control algorithm could be to achieve, during the gearshift, the same level of longitudinal acceleration (perhaps after a short transition) compared to a vehicle with the new gear ratio inserted with the conditions of pedal throttle position currently imposed by the driver. On a vehicle equipped with an automated manual transmission, the driver does not have to release the throttle pedal during the gearshift.

\footnotetext{
The Engineering Meetings Board has approved this paper for publication. It has successfully completed SAE's peer review process under the supervision of the session organizer. This process requires a minimum of three (3) reviews by industry experts.

All rights reserved. No part of this publication may be reproduced, stored in a retrieval system, or transmitted, in any form or by any means, electronic, mechanical, photocopying, recording, or otherwise, without the prior written permission of SAE.

ISSN 0148-7191

Positions and opinions advanced in this paper are those of the author(s) and not necessarily those of SAE. The author is solely responsible for the content of the paper.

SAE Customer Service: Tel: $\quad 877-606-7323$ (inside USA and Canada)

Tel: $\quad$ 724-776-4970 (outside USA)

Fax: $\quad 724-776-0790$

Email: CustomerService@sae.org
}

SAE Web Address: http://www.sae.org 


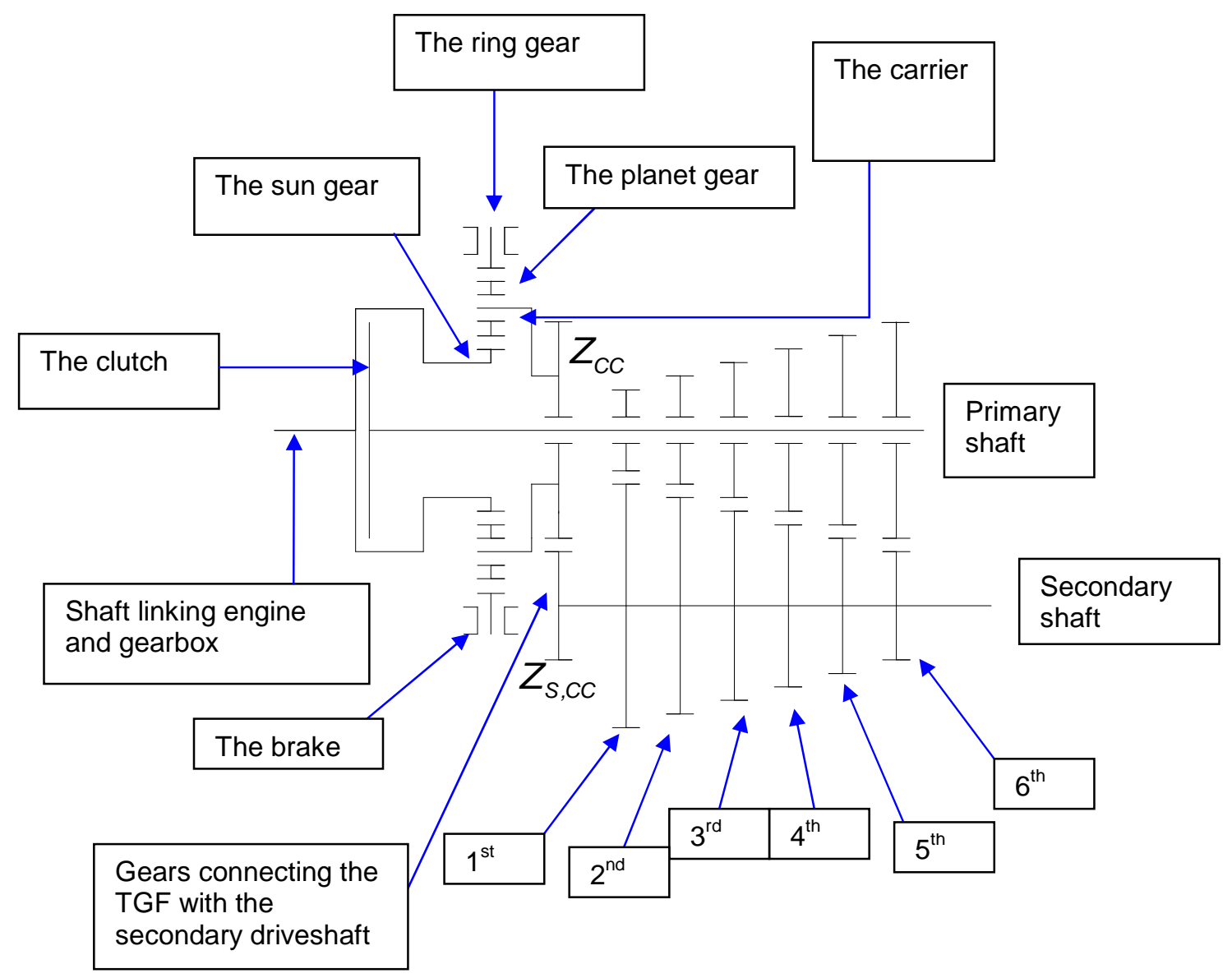

Figure 1 - First possible layout for the implementation of the Torque Gap Filler (TGF Type I)

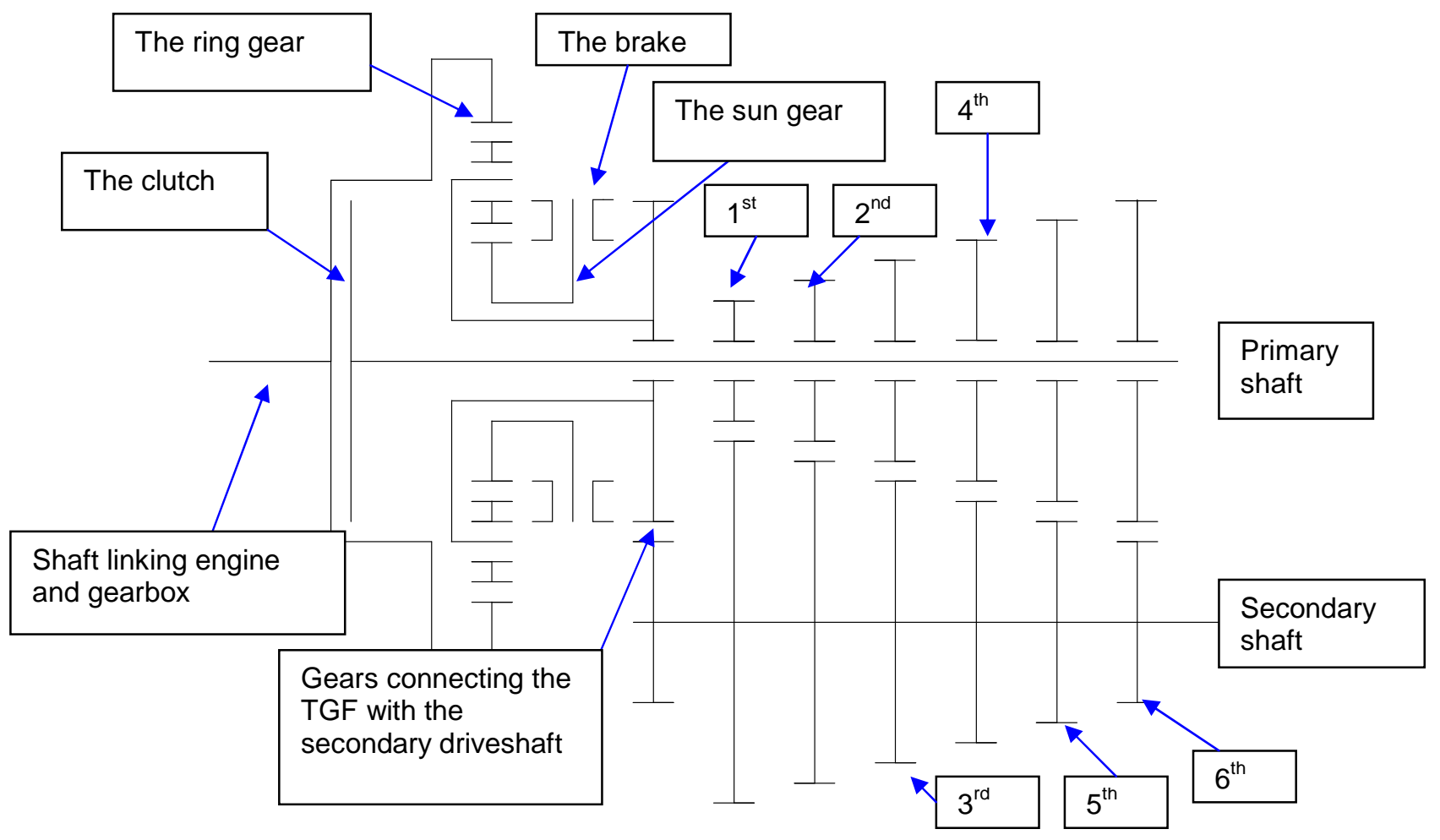

Figure 2 - Second possible layout for the implementation of the Torque Gap Filler (TGF Type II) 
The control algorithm of the TGF has three main outputs, the first one in terms of reference brake torque, the second one in terms of required engine torque and the third one in terms of clutch disc axial displacement (a devoted sensor is usually adopted in the case of an automated manual transmission).

\section{BASIC EQUATIONS GOVERNING THE SYSTEM}

In this section the basic equations for the system are presented under the hypothesis of configuration 1 . These equations can be adopted as the basis for the design of the control algorithm. In a very first approximation, if driveline dynamics are not considered, vehicle inertia can be supposed as if it were directly linked to the secondary shaft of the gearbox. The effect of the clutch damper and half shafts is not considered for this first approximation model devoted to the development of the control algorithm. Under this hypothesis and without considering synchronization dynamics, the power-train of a vehicle equipped with the TGF can work in two different states:

- $\quad$ slipping clutch;

- engaged clutch.

At this step, TGF dynamics are taken into account by considering the inertia $J_{3}$ of the brake disc.

\section{Slipping Clutch}

In this condition, the system can be represented by the following equations:

$T_{\text {engine }}-J_{\text {eng }} \frac{d \omega_{\text {eng }}}{d t}=F_{1} R_{1}+T_{\text {clutch }}$

where $T_{\text {engine }}$ is engine torque, $J_{\text {eng }}$ is engine inertia, $\frac{d \omega_{\text {eng }}}{d t}$ is engine angular acceleration, $F_{1}$ is the force between the teeth of the gears of the epicyclical gearbox (under the hypothesis of unit efficiency), $R_{1}$ is sun gear radius, $T_{\text {clutch }}$ is the torque transmitted by the clutch. Vehicle longitudinal dynamics are given by:

$$
\begin{aligned}
& T_{\text {out }, T G F} \frac{Z_{S, C C}}{Z_{C C}} \rho_{P}-T_{\text {res }}+T_{\text {clutch }} \rho_{C x} \rho_{P}= \\
& =J_{\text {veh }} \frac{d \omega_{\text {veh }}}{d t}
\end{aligned}
$$

where $T_{\text {out,TGF }}$ is the output torque from the carrier of the epicyclical gearbox, $Z_{S, C C}$ is the number of the teeth of the gear on the secondary shaft of the gearbox (Figure $1), Z_{C C}$ is the number of the teeth of the gear on the carrier (Figure 1), $\rho_{P}$ is the final gear ratio at the differential, $T_{\text {res }}$ is the torque due to rolling resistance of the tires, aerodynamic drag and longitudinal slope of the road, $\rho_{C x}$ is the current gear ratio, $J_{v e h}$ is the equivalent inertia of the vehicle and $\frac{d \omega_{v e h}}{d t}$ is wheel angular acceleration. In a first approximation, $T_{\text {out,TGF }}$ can be expressed by:

$$
T_{\text {out }, T G F}=F_{1}\left(R_{1}+R_{3}\right)
$$

where $R_{3}$ is the radius of the ring gear of the TGF. The dynamics of the brake disc and the ring gear is given by:

$$
-F_{1} R_{3}+T_{B R A K E}=J_{3} \frac{d \omega_{3}}{d t}
$$

where $T_{B R A K E}$ is the applied brake torque (according to the control algorithm of the TGF), $J_{3}$ is brake disc and ring gear inertia, $\frac{d \omega_{3}}{d t}$ is ring gear angular acceleration. The Willis formula for epicyclical gearboxes can be applied to this system (where $\Omega$ is the angular velocity of the carrier):

$$
\Omega=\frac{\omega_{1} R_{1}+\omega_{3} R_{3}}{R_{1}+R_{3}}
$$

By combining equations (1)-(5), it is possible to find out the two equations which govern the entire system. The equation which permits the computation of the dynamics of the brake is:

$$
\begin{aligned}
& T_{\text {BRAKE }}\left(\frac{\rho_{P} Z_{S, C C}}{Z_{C C}} \frac{Z_{1}+Z_{3}}{Z_{3}}+\frac{J_{v e h} Z_{C C} Z_{1}^{2}}{Z_{S, C C} J_{3} \rho_{P} Z_{1}\left(Z_{1}+Z_{3}\right)}\right)+ \\
& -T_{\text {engine }} \frac{J_{v e h} Z_{C C} Z_{1}}{Z_{S, C C} J_{1} \rho_{P}\left(Z_{1}+Z_{3}\right)}+ \\
& -T_{\text {res }}+T_{\text {clutch }}\left(\rho_{C X} \rho_{P}+\frac{J_{v e h} Z_{C C} Z_{1}}{J_{1} Z_{S, C C} \rho_{P}\left(Z_{1}+Z_{3}\right)}\right)= \\
& =\frac{d \omega_{3}}{d t}\left(\frac{J_{3} Z_{S, C C} \rho_{P}\left(Z_{1}+Z_{3}\right)}{Z_{3} Z_{C C}}+\frac{J_{v e h} Z_{C C}}{\rho_{P} Z_{S, C C}}\right)
\end{aligned}
$$

Where $Z_{1}$ and $Z_{3}$ are the number of teeth which are proportional to the radii $R_{1}$ and $R_{3}$.

Equation (7) is the dynamic equilibrium of engine driveshaft:

$$
\begin{aligned}
& \frac{T_{\text {engine }}-T_{\text {clutch }}}{J_{\text {eng }}}-T_{\text {BRAKE }} \frac{Z_{1}}{J_{\text {eng }} Z_{3}}+\frac{d \omega_{3}}{d t}\left(\frac{J_{3} Z_{1}}{J_{\text {eng }} Z_{3}}\right)= \\
& =\frac{d \omega_{\text {eng }}}{d t}
\end{aligned}
$$




\section{Engaged Clutch}

The system enters the condition of engaged clutch when the sliding motion between the engine and the clutch ceases. In the case of engaged clutch, the system is characterized by only one degree of freedom (rigid connection between engine, gearbox, wheels and vehicle equivalent inertia). The ratio between the velocity of the carrier of the epicyclical gearbox and the velocity of the engine must be consistent with the current gear ratio $\rho_{C x}$. The following equations express the kinematical link between the different components of the system:

$\omega_{3}=\Omega \frac{Z_{C C} \rho_{C x}}{Z_{S, C C}} \frac{\frac{Z_{S, C C}}{Z_{C C} \rho_{C x}}\left(Z_{1}+Z_{3}\right)-Z_{1}}{Z_{3}}=$
$=\omega_{\text {eng }} \frac{Z_{S, C C}\left(Z_{1}+Z_{3}\right)}{Z_{3}\left(Z_{C C} \rho_{C x}-Z_{1}\right)}$
Eq. (9) can be obtained by re-arranging (8):

$$
\Omega=\frac{\omega_{e n g} Z_{S, C C}}{Z_{C C} \rho_{C X}}
$$

By considering equations (1), (2), (3), (8) and (9), it is possible to obtain the simplified equation (10), which governs the dynamics of the entire system.

This equation is simplified as it does not consider the stiffness and damping properties of the clutch damper and the half shafts, which are responsible for the overall system dynamics.

$T_{\text {BRAKE }}\left[\frac{\left(Z_{1}+Z_{3}\right) Z_{S, C C} \rho_{P}}{Z_{3} Z_{C C}}-\frac{Z_{1} \rho_{C X} \rho_{p}}{Z_{3}}\right]+T_{\text {engine }} \rho_{C X} \rho_{P}-T_{\text {res }}=$

$=\frac{d \Omega}{d t}\left[\frac{J_{3}\left(\frac{Z_{S, C C}}{Z_{C C} \rho_{C X}}\left(Z_{1}+Z_{3}\right)-Z_{1}\right)}{Z_{3}}\left(\left(Z_{1}+Z_{3}\right) \rho_{C x} \rho_{P}-\frac{Z_{C C} \rho_{C x}{ }^{2} \rho_{P} Z_{1}}{Z_{S, C C} Z_{3}}\right)+J_{1} \frac{Z_{C C} \rho_{C X}{ }^{2} \rho_{P}}{Z_{S, C C}}+J_{v e h} \frac{Z_{C C}}{\rho_{P} Z_{S, C C}}\right]$

The system remains in the condition of engaged clutch if the following condition is satisfied:

$T_{\text {clutch, computed }} \leq T_{\text {clutch,MAX }}$

$T_{\text {clutch,MAX }}$ is the maximum torque which can be transmitted by the engaged clutch. It depends on clutch axial displacement and friction coefficient (it is the same torque which is computed in the case of slipping clutch). $T_{\text {clutch,computed }}$ is the torque which is transmitted by the clutch instant by instant when the clutch is engaged. It can be obtained as a function of (1) and the kinematical equations for the case of engaged clutch. As a consequence, $T_{\text {clutch,computed }}$ can be expressed by:

$$
\begin{aligned}
& T_{\text {clutch,computed }}=T_{\text {engine }}-T_{\text {BRAKE }} \frac{Z_{1}}{Z_{3}}+ \\
& +J_{3} \frac{d \Omega}{d t} \frac{Z_{c c} \rho_{c X}}{Z_{s, c c}} \frac{\frac{Z_{s, c c}}{Z_{c c} \rho_{c x}}\left(Z_{1}+Z_{3}\right)-Z_{1}}{Z_{3}}+ \\
& -J_{\text {eng }} \frac{d \Omega}{d t} \frac{Z_{c c} \rho_{c X}}{Z_{s, c c}}
\end{aligned}
$$

\section{CONTROL ALGORITHM}

\section{Basic Concepts}

The basic function of the control algorithm of the TGF consists of eliminating the torque gap during the gearshift. This result can be obtained through a proper control of the brake torque during the gearshift, when the driveline is in the condition of sliding friction. If we neglected the inertial effect due to ring gear and the brake disc of the TGF, (1) and (2) would become uncoupled equations. In particular, (2) would become: 
$T_{\text {BRAKE }} \frac{R_{1}+R_{3}}{R_{3}} \frac{Z_{S, C C}}{Z_{C C}} \rho_{P}-T_{\text {res }}+$

$+T_{\text {clutch }} \rho_{C x} \rho_{P}=J_{\text {veh }} \frac{d \omega_{\text {veh }}}{d t}$

During most of the duration of the intervention of the brake of the TGF, $T_{\text {clutch }}$ is null. The term $T_{\text {BRAKE }} \frac{R_{1}+R_{3}}{R_{3}} \frac{Z_{S, C C}}{Z_{C C}} \rho_{P}$ represents the torque (referred to the wheels) which would be transmitted to the wheels during the gearshift. As a consequence, the reference brake torque for the TGF during the gearshift is given by:

$$
\begin{aligned}
& T_{B R A K E, \text { ref }}=T_{\text {engine }}\left(\% \text { throttle, } \omega_{\text {eng }}\right) . \\
& \cdot \frac{\rho_{C X} R_{3} Z_{C C}}{\left(R_{1}+R_{3}\right) Z_{S, C C}}
\end{aligned}
$$

Where $T_{\text {engine }}\left(\%\right.$ throttle, $\left.\omega_{\text {eng }}\right)$ can be computed as a function of engine map. (14) is an approximated formula since it does not take account of the effect due to engine inertia. In fact, if the considered acceleration maneuver were performed by the vehicle in the condition of inserted gear ratio (the new gear ratio) and not by the vehicle with the TGF working during the gearshift, the real output torque from engine shaft would be reduced as a function of the contribution due to engine shaft acceleration. As a consequence, the second approximation formula in order to compute the reference torque for the brake would be given by:

$$
\begin{aligned}
& T_{B R A K E, \text { ref }}=\left(T_{\text {eng }}\left(\% \text { throttle, } \omega_{\text {eng }}\right)-J_{\text {eng }} \frac{d \omega_{\text {eng }}^{*}}{d t}\right) . \\
& \frac{\rho_{C X} R_{3} Z_{C C}}{\left(R_{1}+R_{3}\right) Z_{S, C C}}
\end{aligned}
$$

where the term $\frac{d \omega_{\text {eng }}^{*}}{d t}$ corresponds to the hypothetical angular acceleration of the engine shaft for the vehicle accelerating according to the new gear ratio in the condition of engaged clutch. $\frac{d \omega_{e n g}^{*}}{d t}$ has nothing in common with the actual $\frac{d \omega_{e n g}}{d t}$. In fact, during TGF interventions, in most cases (during upshifts) it is:

$$
\left(\frac{d \omega_{e n g}^{*}}{d t}>0\right) \text { and }\left(\frac{d \omega_{e n g}}{d t}<0\right)
$$

The adopted brake is typically based on hydraulic actuation. Brake reference pressure $p_{B R A K E \text {,ref }}$ is given by:

$$
p_{B R A K E, r e f}=\frac{T_{B R A K E, r e f}}{A_{B C} r_{e q} B F}
$$

where $A_{B C}$ is brake cylinder area, $r_{e q}$ is equivalent disc radius, $B F$ is the brake factor, the ratio between brake drag force and brake clamping force.

The most challenging obstacle in actuating the presented feedforward control algorithm for TGF brake is due to the fact that the actuation system of the TGF can be equipped (for reasons of cost) only with a pressure sensor (and not a torque sensor). As a consequence, it is possible a feedback (and precise) actuation only from the point of view of pressure. A significant limit is due to the fact that $B F$ is a function of brake pressure $p$, brake temperature $T$ (on the contact surfaces) and brake angular speed $\omega_{3}$. Since $p$ and $\omega_{3}$ can be measured on the real plant, brake temperature $T$ should be estimated by implementing, within the control algorithm, the typical equation for convective heat exchange (the author has acquired a considerable experience in the field of clutch temperature estimation, [5], [12]):

$$
\begin{aligned}
& T_{\text {BRAKE, estimated }} \cdot \omega_{3}+h\left(T_{\text {ambient }}-T\right)= \\
& =C_{\text {BRAKE }} \frac{d T}{d t}
\end{aligned}
$$

$T_{\text {BRAKE,estimated }}$ is the estimated brake temperature, $h$ is convective thermal coefficient, $T_{\text {ambient }}$ is air temperature around the brake, $C_{B R A K E}$ is brake disc thermal capacity. From a practical point of view, $h$ can be estimated on the basis of experimental tests (it will be a function of brake disc speed), $T_{\text {ambient }}$ can be deducted as a function of engine temperature. The last comments explain how difficult the real-world implementation of the presented feedforward control algorithm can be. As a consequence, it is necessary to implement a feedback control algorithm, which, on the difference between the desired level average level and the obtained level of longitudinal acceleration during the gearshift, computes a corrective coefficient, which is progressively modified after each intervention of the TGF. This coefficient is a function of the behavior of the system during a pre-defined number $N$ of gearshifts characterized by the use of the TGF. From a theoretical point of view, it would be possible to implement directly and exclusively a feedback control algorithm, based on the real time comparison of the desired and the obtained level of longitudinal acceleration of the vehicle. However, from a practical point of view, several experimental tests carried out during the development of automated manual transmission systems demonstrated the poor results 
deriving from this solution. When, during the gearshift, the clutch is slipping and it is transmitting a torque, it is necessary to take account its contribution when computing TGF brake reference pressure:

$$
\begin{aligned}
& p_{B R A K E, \text { ref }}= \\
& =\left(T_{\text {engine }}\left(\% \text { throttle, } \omega_{\text {eng }}\right)-T_{\text {clutch }}-J_{\text {eng }} \frac{d \omega_{\text {eng }}^{*}}{d t}\right) . \\
& \cdot \frac{\rho_{C X} R_{3} Z_{C C}}{\left(R_{1}+R_{3}\right) Z_{S, C C} A_{B C} r_{e q} B F}
\end{aligned}
$$

The time history of $T_{\text {clutch }}$ (related to its axial displacement) is directly determined by the control algorithm of the automated manual transmission system, and affects the TGF control through equation (19). Under the same basic hypothesis under which equation (13) was found out, it is possible to write:

$$
T_{\text {engine }}-J_{\text {eng }} \frac{d \omega_{\text {eng }}}{d t}=T_{\text {BRAKE }} \frac{R_{1}}{R_{3}}+T_{\text {clutch }}
$$

Equation (20) shows that engine dynamics during the gearshift (in a first approximation, we can neglect the term $T_{\text {clutch }}$ ) depend both on $T_{\text {engine }}$ and $T_{\text {BRAKE }}$. Since $T_{B R A K E}$ is the independent variable (it determines vehicle longitudinal dynamics, which is the target of TGF), it is necessary to compute $T_{\text {engine }}$ in order to have the desired engine dynamics. In this case, a feedback Proportional Integrative Derivative (PID) controller based on engine angular speed can be an effective solution. During a gearshift, engine speed is subjected to a transition from the initial value $\omega_{v e h}(t) \rho_{P} \rho_{C \text {, old }}$ to a final value $\omega_{\text {veh }}(t) \rho_{P} \rho_{C \text {,new }}$. It must be observed that the value of $\omega_{\text {veh }}(t)$ after the gearshift is different from the value at the beginning of the gearshift. The controller gives origin to a time history of the reference profile of an adimensionalized velocity factor $K_{\text {eng }}$ during the intervention of TGF. The adimensionalization permits to use the same reference profile during all the gearshifts. The reference value of the adimensionalized parameter is equal to 1 at the beginning of the gearshift (at time $t=0$ ) and becomes equal to $\frac{\rho_{C, \text { new }}}{\rho_{C \text {,old }}}$ at the end of the gearshift (at the instant $t=t_{\text {final }}$ ), where $\rho_{C \text {,new }}$ and $\rho_{C, \text { old }}$ are the gear ratios after and before the gearshift. The control algorithm, depending on the driver's preference and on the conditions of the engine and the vehicle, can choose the value of $t_{\text {final }}$ and also the profile for the reduction of engine speed (Figure 3). The reference value (to be sent to the PID controller) of engine speed during the gearshift is given by:

$$
\omega_{\text {eng, reference }}=\omega_{\text {veh }}(t) \rho_{P} \rho_{C, \text { old }} K_{\text {eng }}
$$

For system Type II, the brake torque equation (equivalent of Eq. (15)) would be:

$$
\begin{aligned}
& T_{\text {BRAKE }}=\left[T_{\text {engine }}\left(\% \text { throttle }, \omega_{\text {eng }}\right)-J_{\text {eng }} \frac{d \omega_{\text {eng }}^{*}}{d t}\right] . \\
& \cdot \rho_{C X} \frac{1}{\rho_{C / S S}} \cdot \frac{R_{1}}{R_{1}+R_{3}}
\end{aligned}
$$

Where $\rho_{C / S S}=\frac{Z_{S, C C}}{Z_{C C}}$.

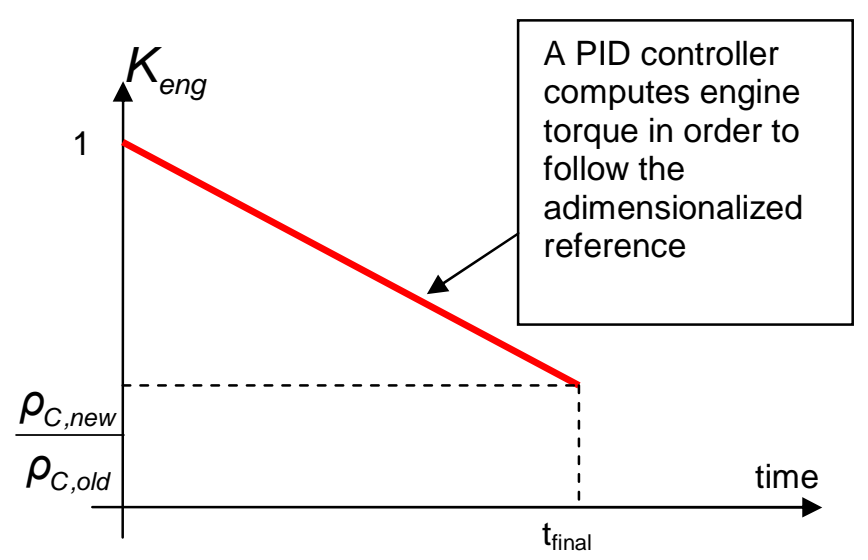

Figure 3 - Example of time history of $K_{\text {eng }}$ during a gearshift. An upshift is assumed as this is the condition for the interventions of the TGF

\section{LIMITATIONS RELATED TO THE SYSTEM LAYOUT}

This section of the article deals with the limitations of the overall system performance induced by its hardware layout.

TGF can transmit torque to the secondary shaft of the gearbox through the brake only if the brake rotates in the opposite direction to the engine shaft. This can only happen if the gear ratio of the epicyclical gearbox is arranged so that, when the ring gear is locked, the gearbox is characterized by a lower torque multiplication than that the ratio due to the current gear ratio. If the sense of the rotation of the disc and the engine shaft were the same, the brake torque would reduce vehicle speed, which is not the main purpose of TGF (it could be marginally useful during downshifts). On the other side, the target of TGF has to be achieved by a system having physical dimensions compatible with its mounting inside the clutch bell of a conventional manual transmission.

With regard to a Type I TGF system, the ring gear must rotate in a direction opposite to that of the engine in order for the system to function as required. If the ring gear and the engine are rotating in the same direction, any braking torque applied will lead to an increase in engine 
speed, and a deceleration of the vehicle. It is therefore of great importance to know the point at which the TGF system will cease to function.

This can be achieved by using the Willis Formula. At the moment in time at which the rotations of the ring gear and the engine become unidirectional, the speed of the ring can be assumed to equal zero, thus the Willis equation becomes:

$\Omega=\frac{\Omega_{e n g} Z_{1}}{Z_{1}+Z_{3}}$

By equating (9) and (23), it is:

$\frac{\Omega_{e n g} Z_{1}}{Z_{1}+Z_{3}}=\frac{\Omega_{E} \rho_{C / S S}}{\rho_{C i}}$

which can then be simplified and rearranged to give the minimum gear ratio $\rho_{C i}$ at which the TGF still works:

$\rho_{C i}=\frac{\rho_{C / S S}\left(Z_{1}+Z_{3}\right)}{Z_{1}}$

When the above expression is true, the ring gear has an angular velocity equal to zero. In order for the TGF system to increase longitudinal vehicle acceleration during a gearshift, the following expression must be satisfied:

$\rho_{C i}>\frac{\rho_{C / S S}\left(Z_{1}+Z_{3}\right)}{Z_{1}}$

Note that in order for the TGF to operate, the term on the right hand side of Eq. (26), must be smaller than the gear ratio in which the system is required to function. Note that the direction of the inequality symbol is a result of the higher gears of the vehicle being smaller values (i.e. the gear ratio of $1^{\text {st }}$ gear is a larger number than the gear ratio of $6^{\text {th }}$ gear).

In a similar way, for the second layout the equivalent of (26) is:

$\rho_{C i}>\frac{\rho_{C / S S}\left(Z_{1}+Z_{3}\right)}{Z_{3}}$

\section{RESULTS}

From Eq. (14) it is possible to calculate the required level of brake torque for a given system of the Type I variant. When making a comparison between two systems that work in a different number of gears, the majority of parameters in the equations are the same for both systems. The only term that does vary is the final term; the $Z$ ratios and possibly $\rho_{C / s S}$. For example, in order to discover the brake torque ratio between a system that employs the TGF in 'i' gearshifts (refer to the subscript 'i' in eq. (28)), and one that only employs the system for the gearshift from $1^{\text {st }}$ to $2^{\text {nd }}$ gear (for which it is: $\rho_{C i}=\rho_{C 2}$, no subscript in eq. (28)), it can be written that:

$$
\frac{T_{\text {BRAKE }(i)}}{T_{\text {BRAKE (1GS) }}}=\frac{\frac{Z_{3(i)}}{\rho_{C / S S(i)}\left(Z_{3(i)}+Z_{1(i)}\right)}}{\frac{Z_{3}}{\rho_{C / S S}\left(Z_{3}+Z_{1}\right)}}
$$

Similarly, it is possible to calculate the ratio between the minimum gear ratio $\rho_{C i}$ in which the TGF will operate and $\rho_{C 2}$ :

$\frac{\rho_{C i}}{\rho_{C 2}}=\frac{\frac{\rho_{C / S S(i)}\left(Z_{1(i)}+Z_{3(i)}\right)}{Z_{1(i)}}}{\frac{\rho_{C / S S}\left(Z_{1}+Z_{3}\right)}{Z_{1}}}$

With Equations (28) and (29) as a foundation, it becomes possible to develop an expression that relates the brake torque ratio between two systems, to the number of gears in which the TGF is required to operate in.

$$
\frac{T_{B R A K E(i)}}{T_{B R A K E(1 G S)}}=\frac{1}{k_{1}}\left(\frac{1}{\rho_{C / S S(i)}}-\frac{1}{k_{2} \frac{\rho_{C i}}{\rho_{C 2}}}\right)
$$

Where:

$$
\begin{aligned}
& k_{1}=\frac{Z_{1}}{\rho_{C / S S}\left(Z_{3}+Z_{1}\right)} \\
& k_{2}=\frac{\rho_{C / S S}\left(Z_{1}+Z_{3}\right)}{Z_{3}}
\end{aligned}
$$

Similar calculations can be carried out for the computation of the velocity of the brake and the power dissipated within the brake as functions of the adimensionalized gear ratio $\frac{\rho_{C i}}{\rho_{C 2}}$ (it is the parameter Rho Ci / Rho C2 on the horizontal axes of the graphs in Figures 4-8). The system which works at lower levels of torque and power for the same number of upshifts is the better one. 


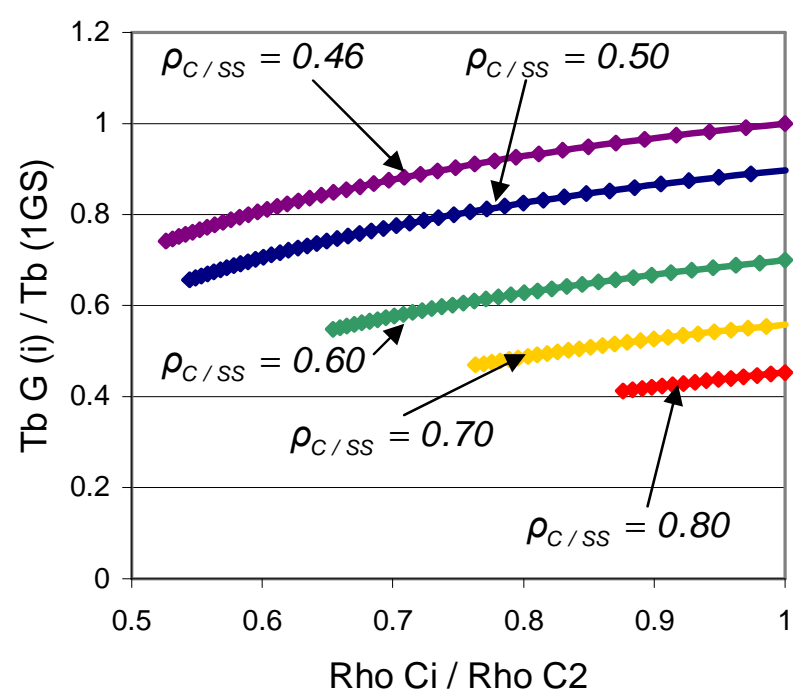

Figure 4 - Brake torque ratio as a function of the adimensionalized minimum gear ratio at which TGF can operate (TGF Type I)

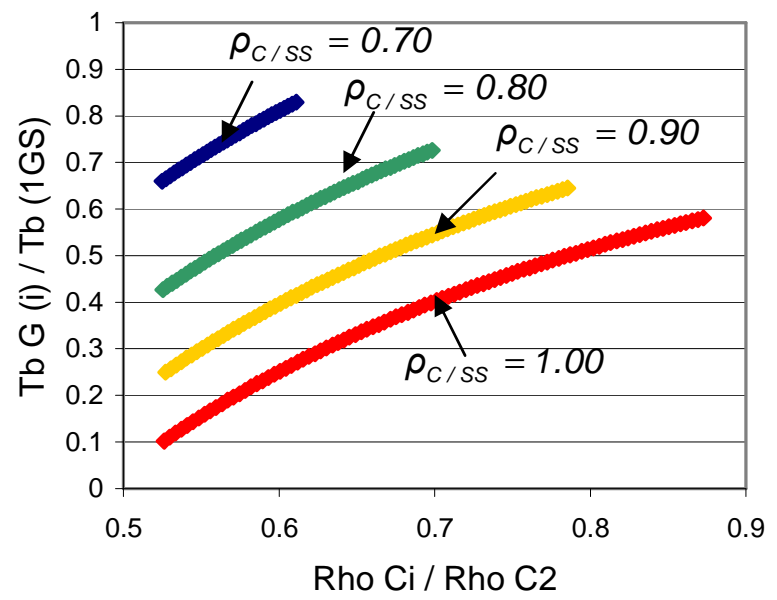

Figure 5 - Brake torque ratio as a function of the adimensionalized minimum gear ratio at which TGF can operate (TGF Type II)

Figures 4 and 5 show the brake torque ratio as a function of the maximum adimensionalized gear ratio at which TGF can operate (respectively TGF Type I and II). Figure 6 shows the analysis in terms of angular velocity of the brake for TGF Type I. Figures 7 and 8 show the comparison between Type I and Type II in terms of dissipated power within the brake. Figure 8 shows the same trend as the Type I system, but it should be noted that the power ratio is confined to a smaller range. This is a real advantage of the Type II system since it indicates that the difference in heat generated at the brake for a given gearshift, between a system operating in all gears, and a system operating in just one gearshift is not as significant as for a Type I system.

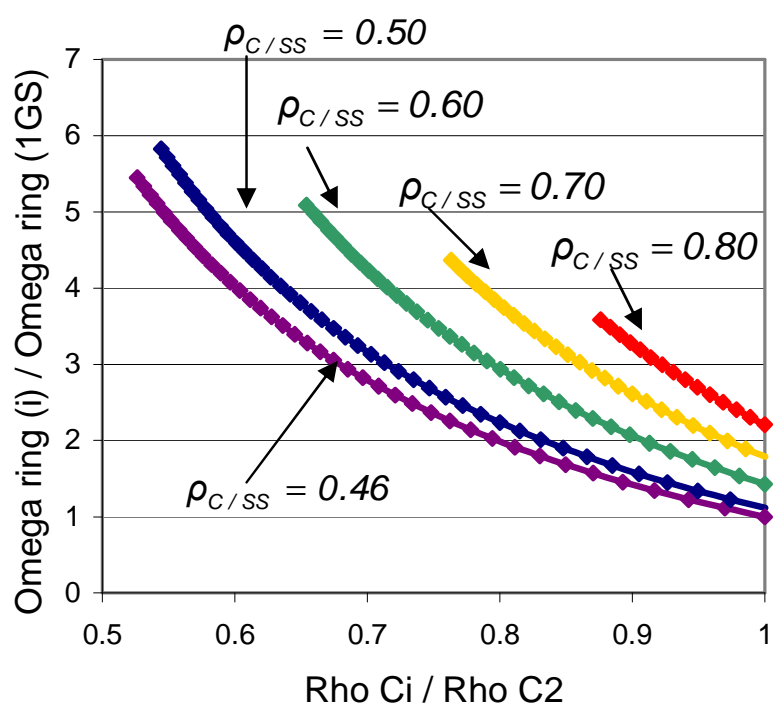

Figure 6 - Ring speed ratio (between the ring speed of a TGF system which can work for a maximum of $i$ upshifts and a system which can work for one upshift only) as a function of the adimensionalized maximum gear ratio at which TGF can operate (TGF Type I)

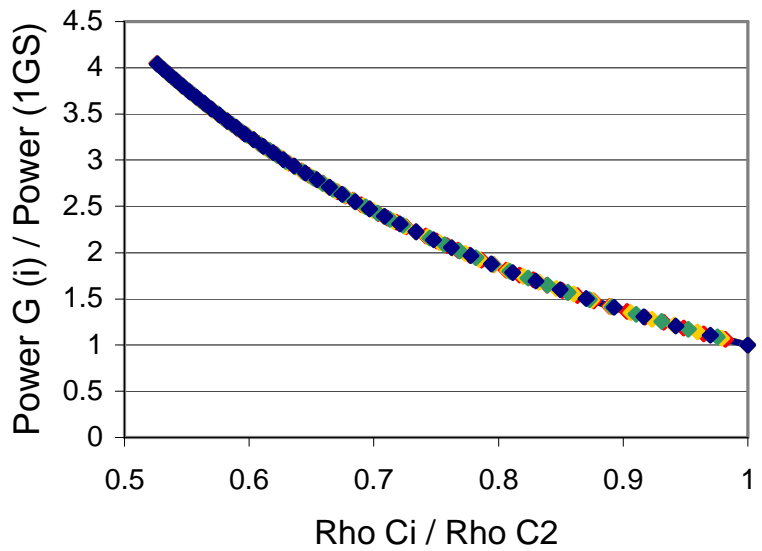

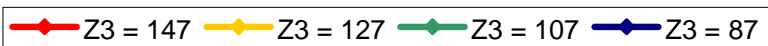

Figure 7 - Brake power ratio (between the brake power of a TGF system which can work for a minimum gear ratio $\rho_{C i}$ and a system which can work for one upshift only) as a function of the adimensionalized minimum gear ratio at which TGF can operate (TGF Type I)

A complete simulator of the system has been developed [10], including synchronization dynamics and the actuation delays of the TGF. Figure 9 shows the angular velocities of the different components of the system (TGF Type I) during a maneuver consisting of a series of upshifts. The system adopted in this simulation works during the first two gearshifts, as in the third gear the velocity of the brake disc is equal to zero. 


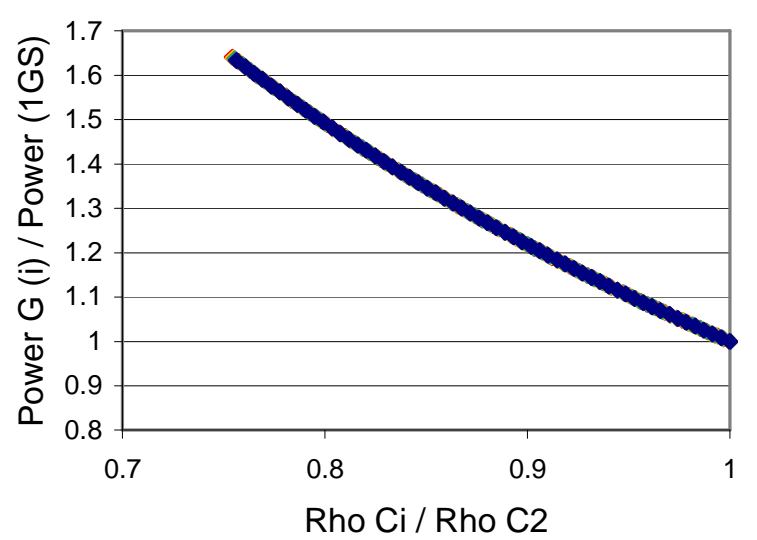

$\smile$ Z3 = $190 \longleftarrow$ Z3 = $170 \multimap$ Z3 = $150 \multimap$ Z3 = 130

Figure 8 - Brake power ratio (between the brake power of a TGF system which can work for a maximum of $\mathrm{i}$ gear ratios and a system which can work for one upshift only) as a function of the adimensionalized minimum gear ratio at which TGF can operate (TGF Type II)
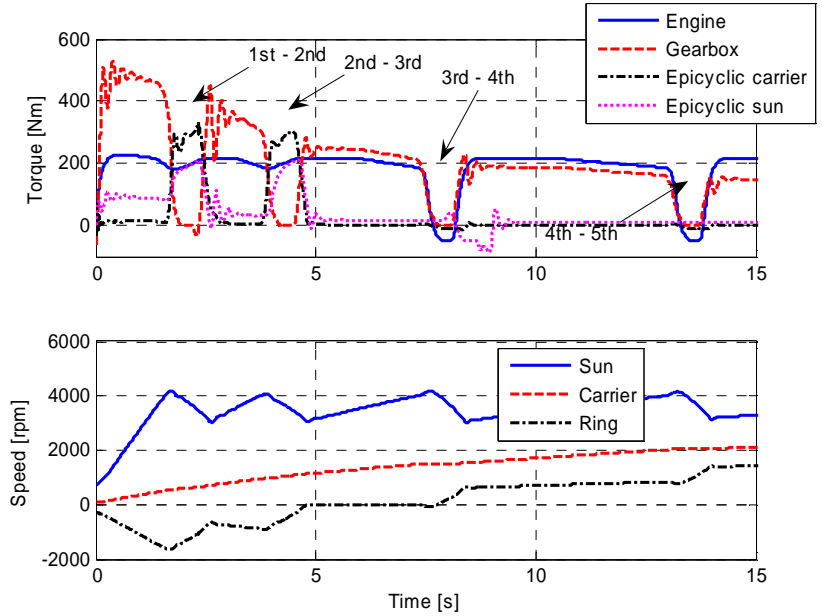

Figure 9 - Time histories of the angular velocities of the components of the TGF during a sequence of gearshifts (TGF active in the gearshifts $1 \rightarrow 2$ and $2 \rightarrow 3$ )

Figure 10 shows the detailed time histories of brake torque and speed during the upshifts $1 \rightarrow 2$ and $2 \rightarrow 3$. Figure 11 shows a comparison, within a longitudinal acceleration test, between a vehicle equipped with a manual transmission system and the same vehicle equipped with an automated manual transmission system with TGF device.

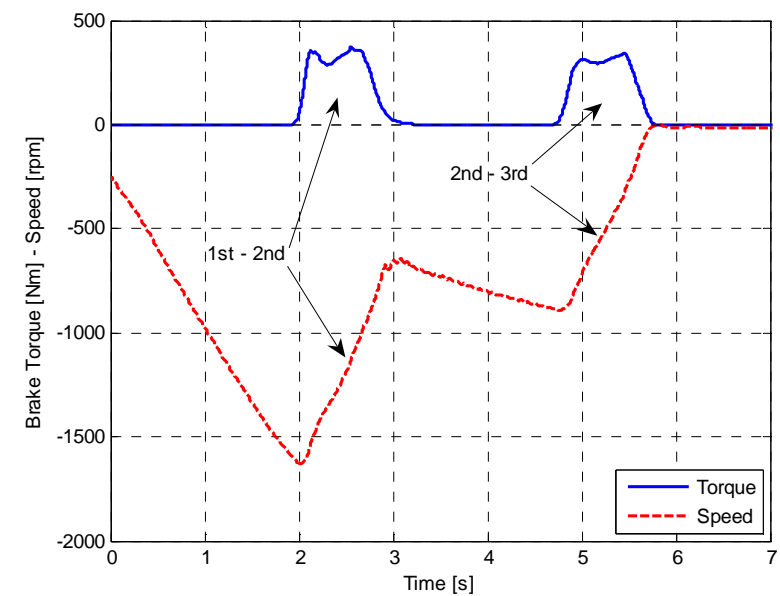

Figure 10 - Braking torque and angular velocity of the TGF ring gear
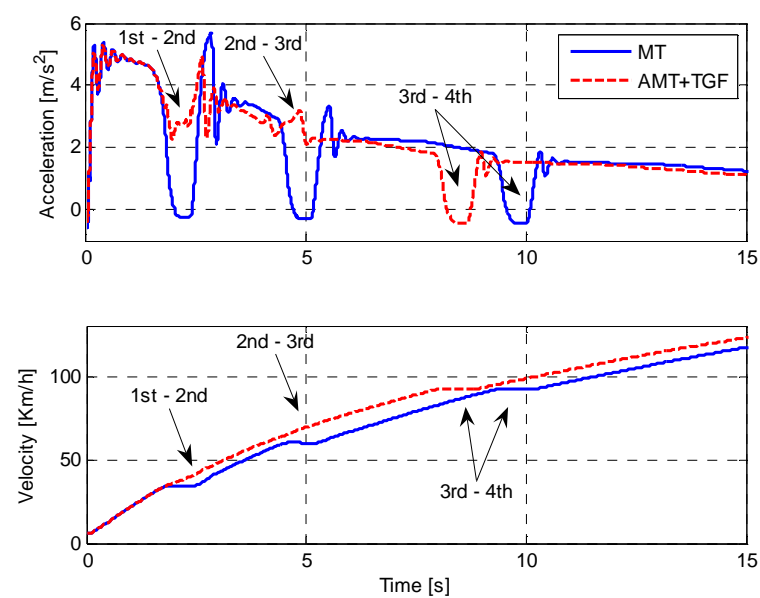

Figure 11 - Acceleration and velocity comparison between a manual transmission and an automated manual transmission with TGF device

\section{CONCLUSION}

The basic principles for TGF conception and design have been explained in detail. In particular, a physical explanation of the working principle of the two possible different layouts of the system has been dealt with. The equations for the implementation of the control algorithm for the two alternative layouts have been obtained. The description of a possible control algorithm for the system has been diffusely carried out. Finally, a quantitative and objective comparison of the performance of the two possible layouts of the system has been discussed by using adimensionalized diagrams. An experimental activity for the physical testing of the system and the implementation of the discussed control algorithms is going to be carried out.

\section{REFERENCES}

1. S. Sehyun, H. Chang, I. Joo, 'Effect of Scroll Angle on Performance of Automotive Torque Converter', SAE 2000-01-1158; 
2. G. Kim , J. Jae-Duk, 'Effects of Stator Shapes on Hydraulic Performances of An Automotive Torque Converter With a Squashed Torus', SAE 2002-010886;

3. S. A. Edelen, J. Morscheck, 'Automated Mechanical Transmissions', SAE 973176;

4. W. Hardtle, 'New Automated Mechanical Transmission-Customer Considerations, Concept Comparisons and Experiences', SAE 982796;

5. A. Sorniotti, N. D'Alfio, E. Galvagno, A. Morgando, F. Amisano, 'Hardware-In-the-Loop Testing of Automotive Control Systems', SAE 2006-01-1962;

6. A. Sorniotti, 'Driveline Modeling, Experimental Validation and Evaluation of the Influence of the Different Parameters on the Overall System Dynamics', SAE 2008-01-0632;

7. B. Matthes, F. Guenter, 'Dual Clutch Transmissions Lessons Learned and Future Potential', SAE 200501-1021;

8. X. Song, D. G. Smedley, 'Simulation Study of Dual Clutch Transmission for Medium-Duty Truck Applications', SAE 2005-01-3590;

9. J.C. Wheals, A. Turner, K. Ramsay, A. O'Neill, J. Bennett, H. Fang, 'Double Clutch Transmission
(DCT) using Multiplexed Linear Actuation Technology and Dry Clutches for High Efficiency and Low Cost', SAE 2007-01-1096;

10. M. Velardocchia, E. Bonisoli, A. Sorniotti, E. Galvagno, N. D'Alfio, 'Torque Gap Filler for Automated Manual Transmissions', SAE 2008-010631;

11. F. Amisano, A. Sorniotti, M. Velardocchia, 'A Method for Controlling a Power Assisted Propulsion System in a Motor Vehicle', European Patent 1921349 A1, Applicant: Magneti Marelli Powertrain SpA;

12. F. Amisano, G. Ercole, G. Mattiazzo, S. Mauro, M. Velardocchia, 'Dynamics of thermal phenomena in diaphragm spring clutch', SAE 1999-25-0210.

\section{CONTACT}

Aldo Sorniotti

University of Surrey

Faculty of Engineering and Physical Sciences

Guildford

Surrey, UK

GU2 7XH

Email: a.sorniotti@surrey.ac.uk 